\title{
A Close Eye on the Eagle-Eyed Visual Acuity Hypothesis of Autism
}

\author{
Sven Bölte • Sabine Schlitt • Volker Gapp • Daniela Hainz • \\ Shella Schirman · Fritz Poustka • Bernhard Weber • \\ Christine Freitag $\cdot$ Angela Ciaramidaro $\cdot$ Henrik Walter
}

Published online: 10 June 2011

(C) The Author(s) 2011. This article is published with open access at Springerlink.com

\begin{abstract}
Autism spectrum disorders (ASD) have been associated with sensory hypersensitivity. A recent study reported visual acuity (VA) in ASD in the region reported for birds of prey. The validity of the results was subsequently doubted. This study examined VA in 34 individuals with ASD, 16 with schizophrenia (SCH), and 26 typically developing (TYP). Participants with ASD did not show higher VA than those with SCH and TYP. There were no substantial correlations of VA with clinical severity in ASD or SCH. This study could not confirm the eagle-eyed acuity hypothesis of ASD, or find evidence for a connection of VA and clinical phenotypes. Research needs
\end{abstract}

S. Bölte $(\bowtie)$

Department of Women's and Children's Health, Astrid Lindgren

Children's Hospital, Q2:07, Center of Neurodevelopmental

Disorders (KIND), Karolinska Institutet, 17176 Stockholm,

Sweden

e-mail: sven.bolte@ki.se

\section{S. Bölte}

Department of Child and Adolescent Psychiatry and

Psychotherapy, Central Institute of Mental Health, Mannheim,

Germany

S. Bölte $\cdot$ S. Schlitt - D. Hainz $\cdot$ S. Schirman · F. Poustka ·

C. Freitag

Department of Child and Adolescent Psychiatry,

Psychosomatics, and Psychotherapy, Goethe-University,

Frankfurt/M., Germany

V. Gapp · B. Weber

Department of Psychiatry, Goethe-University, Frankfurt/M., Germany

\author{
A. Ciaramidaro \\ Center for Cognitive Science, University of Turin, Turin, Italy \\ H. Walter \\ Department of Psychiatry, Charité, Berlin, Germany
}

to further address the origins and circumstances associated with altered sensory or perceptual processing in ASD.

Keywords Autism - Schizophrenia - Vision - Attention · Sensory processing $\cdot$ Bottom-up

\section{Introduction}

The clinical phenotype of autism spectrum disorders (ASD) is defined by impairments in reciprocal social interaction, mutual communication alongside with inflexible behavior patterns and interests. The cognitive phenotype of ASD has not yet been defined, but there is converging evidence that it is characterized by difficulties in social cognition (e.g. Theory of Mind), certain executive malfunctions and attention to detail (ATD), the latter also being known as weak central coherence or local orientation bias (Hill and Frith 2003). ATD refers to a perceptual style characterized by a preference for piecemeal information processing and a decreased drive for good gestalt (Dakin and Frith 2005; Happé and Frith 2006). It is presumed that ATD underlies specific strengths on visual search and spatial tasks exhibited by individuals with ASD, such as superior performance on the Block Design Test (Caron et al. 2006) and the Embedded Figures Test (Shah and Frith 1983), or resisting the influence of context in visual illusions (Happé 1996; Bölte et al. 2007). ATD has also been linked to savant talents (Pring 2005).

The cognitive core of ATD in ASD might be connected to alterations in stimulus-driven/bottom-up versus experience-driven/top-down information processing (Greenaway and Plaisted 2005; Neumann et al. 2006), with ATD in ASD being an epiphenomen of increased bottom-up cognition. On the biological level, neural disconnectivity or 
overconnectivity (Belmonte and Yurgelun-Todd 2003; Geschwind and Levitt 2007) and enhanced perceptual functioning and discrimination (Mottron et al. 2006; Plaisted 2001) have been postulated to cause ATD. While the connectivity approach largely indicates that ATD is a result of a processing deficit, enhanced perceptual functioning and discrimination understands ATD as being based on a processing style.

Both of these general hypotheses for ATD in ASD appear clinically plausible. Lacking neural connectivity or overconnectivity could be associated with multitasking problems, missing the whole picture in complex situations, and problems in language comprehension (Jolliffe and Baron-Cohen 1999; Mackinlay et al. 2006) Enhanced perceptual function could be related to sensory abnormalities. It is widely assumed that sensory alterations and repetitive, stereotypic behavior patterns are closely associated in ASD. Already Leo Kanner described sensory symptoms in seven of his eleven cases. In fact, it is planned that DSM-V criteria for autism spectrum disorder will include sensory processing alterations among the symptoms in stereotypic, repetitive behavior domain (see www.dsm5.org, for details). Anecdotal accounts and questionnaire data support the notion of sensory processing alterations being a core feature of ASD (e.g. Grandin 2000; Tomchek and Dunn 2007). Hypersensitivity in different sensory domains has been repeatedly found (Baron-Cohen et al. 2009). For instance, superior pitch discrimination has been replicated by more than 10 experimental perceptual studies of different groups (e.g. Bonnel et al. 2003; Heaton et al. 2008). In the visual domain, aside from strengths on visual search and spatial tasks mentioned earlier, altered motion processing (possibly reflecting magnocellular malfunction) and typical from processing (possibly reflecting intact parvocellular function) have been discussed (e.g. Bertone et al. 2005; Sutherland and Crewther 2010). A recent article by Ashwin et al. (2009a) examined visual acuity (VA) thresholds in ASD (high-functioning autism, Asperger's syndrome) to test whether visual ATD might be secondary to visual hypersensitivity in terms of altered VA thresholds. The authors found better VA in autism compared to controls-VA so superior that it lay in the region reported for birds of prey ("eagle-eyed VA"). Therefore, it was concluded that sensory thresholds may be informative for research into the aetiology of ASD, and that visual hyperacuity could be a direct precursor of ATD in ASD. The authors discussed that an increased density of foveal cone cells or higher amounts of dopamine receptors at the retinal level might account for their findings.

Ashwin et al.'s article was subsequently commented on by Bach and Dakin (2009) as well as Crewther and Sutherland (2009). They raised methodological doubts about the appropriateness of the technical procedures when measuring VA with the Freiburg Visual Acuity (and Contrast) Test (FrACT) (Bach 1996), a free computer program that uses psychometric methods combined with anti-aliasing and dithering to provide automated, self-paced measurement of visual acuity (www.michaelbach.de/fract/ download.html). The critique points to an unfortunate modification of FrACT default settings, which, according to the comments, made it impossible to measure high thresholds of VA. Instead, low range VA values were extrapolated and resulted in artificially high estimates of high VA thresholds. Typically, a (decimal) VA of 1.0 is assumed to be normal (with increasing values indicating better vision) although young adults usually have a somewhat higher acuity [a median decimal acuity of 1.6 was found in (Rassow et al. 1990)]. The maximum size of measurable decimal VA in Ashwin et al. (2009a) was .43, but reported extrapolated decimal VA values exceed 3.0.

According to the commentaries, a particular problem is that the statistical model used to extrapolate the raw scores (post-hoc maximum likelihood analysis) is highly sensitive to the number of errors made by the participants when judging Landolt Cs, also known as Landolt rings or Landolt broken rings. It is a very commonly used optotype, i.e. a standardized symbol used for testing vision. The Landolt C consists of a ring that has a gap, thus looking similar to the letter C. The gap can be at various positions (usually left, right, bottom, top and the $45^{\circ}$ positions in between) and the task of the tested person is to decide on which side the gap is.

In Ashwin et al. many trials (150) of rather easy to solve Landolt $\mathrm{C}$ stimuli were presented. Therefore, the results could have emerged simply because participants with ASD made a few less errors on these easy stimuli (not difficult ones), owing to better local stimulus attention and more concentration lapses in the typically developing controls, respectively. Thus, the combination of low observation distance, extrapolation of raw data and a high number of trials could have lead to a chain of events causing artifactual VA group differences, leading to the false hypothesis of eagle-eyed vision in autism.

In their reply, Ashwin et al. (2009b) explained their intent when making changes to the FrACT defaults and acknowledge that a chain of unrelated events may have affected the magnitude of the VA measures obtained, but not the existence of the difference between individuals with ASD and controls. They argue that both groups were tested under the same settings in randomized order and that the FrACT is obviously blind to diagnosis. In addition, other studies are cited as complementary evidence for enhanced sensory perception in ASD (Ashwin et al. 2009c; Tavassoli et al. 2009). Nevertheless, the authors agreed that owing to the technical issues outlined by Bach and Dakin (2009) and Crewther and Sutherland (2009) an independent corroboration of their findings would be compulsory. 
The objective of the present study was to contribute such independent VA data from a larger and well characterized sample of individuals with ASD and typically developing controls (TYP), also using the FrACT (with default settings, except for viewing distance). Participants in this study are younger (adolescents/young adults) and comparable for age, which was not the case in the original study, which included ASD participants being considerably older on average (39.9 y) than TYP (27.6 y). Moreover, the current study included females and a clinical control sample of individuals with schizophrenia ( $\mathrm{SCH}$ ), a disorder psychopathologically distinct from ASD (Kolvin 1971; Sporn et al. 2004), but showing clinical and biological links to ASD (Couture et al. 2010; Guilmatre et al. 2009; Magnée et al. 2009; Rapoport et al. 2009). Schizophrenia has an excellent fit as a control group for research in ASD to avoid findings lacking specificity (e.g. Seltzer et al. 2004). It is of particular relevance for the present study, that, as in ASD, the role of sensory processes in the visual domain, altered top-down and bottom-up mechanisms as well as lacking neural connectivity are discussed as pathological mechanisms in $\mathrm{SCH}$ (Hancock et al. 2008; Ribolsi et al. 2009). However, contrary to ASD, rather inferior performance on visual search tasks and decreased sensory functioning has been reported in SCH (Johnson et al. 2005; Yeap et al. 2006). Thus, with regard to the implicit logic of the eagle-eyed VA hypothesis of ASD, one might expect an even larger VA advantage of people with ASD compared to SCH than compared to TYP. However, this study did not hypothesize differences between ASD, SCH and TYP regarding VA.

\section{Methods}

\section{Participants}

The study comprised $n=34$ adolescent and young adult participants with idiopathic high-functioning ASD, $n=16$ with $\mathrm{SCH}$ and $n=26 \mathrm{TYP}(N=76)$ (see descriptive data in Table 1). Eight additional participants (four AUT, three $\mathrm{SCH}$, and one TYP) had been excluded from the starting sample due to incomplete data sets. Inclusion criteria for all participants were: age 15-30 years, IQ $>70$. Additionally, for ASD: an ICD-10 diagnosis F84.0, F84.1 or F84.5; for SCH: an ICD-10 diagnosis F20.0 with at least two clear episodes with paranoid symptoms; for both ASD and SCH: a stable medication 2 weeks prior to participation. Exclusion criteria were for ASD and SCH: presence of any other primary axis I diagnosis, any genetic syndrome, dysmorphic features, and definite neurological disorders e.g. epilepsy). Exclusion criteria for TYP: any neurological or psychiatric disorder. Diagnoses of ASD and $\mathrm{SCH}$ were ICD-10 based clinical consensus classifications of autism $(n=13)$, atypical autism/PDD-NOS $(n=8)$, Asperger's syndrome $(n=13)$ and paranoid schizophrenia, respectively. ASD diagnoses were corroborated by the German versions of the Autism Diagnostic Interview-Revised (ADI-R) and the Autism Diagnostic Observation Schedule (ADOS) (Bölte et al. 2006; Rühl et al. 2004). A diagnosis of Asperger syndrome was given, if the ADI-R autism algorithm cut-off was met, but no history of language delay was apparent on the items first words/first phrases, and

Table 1 Sample characteristics and visual acuity values in the Autism Spectrum Disorders (ASD), Schizophrenia (SCH), and typically developing (TYP) sample

\begin{tabular}{|c|c|c|c|c|c|c|}
\hline $\mathbf{N}$ & $\begin{array}{l}\text { ASD } \\
34 \\
13 \text { AUT, } 13 \text { AS, } 8 \text { AA }\end{array}$ & $\begin{array}{l}\mathrm{SCH} \\
16\end{array}$ & $\begin{array}{l}\text { TYP } \\
26\end{array}$ & $\chi^{2}$ & & $p$ \\
\hline Sex (female/male) & $4 / 30$ & $4 / 12$ & $4 / 22$ & 2.7 & & .31 \\
\hline \multirow{2}{*}{$\begin{array}{l}\text { Handedness } \\
\text { (right/bilateral/left) }\end{array}$} & $29 / 3 / 2$ & $14 / 1 / 1$ & $25 / 0 / 1$ & 3.6 & & .50 \\
\hline & $M( \pm \mathrm{SD})$ & $M( \pm \mathrm{SD})$ & $M( \pm \mathrm{SD})$ & $F^{*}$ & $p$ & $\eta^{2}$ \\
\hline Age (years) & $19.8(4.7)$ & $22.7(5.0)$ & $20.1(3.6)$ & $6.6^{1}$ & .004 & .14 \\
\hline $\begin{array}{l}\text { Nonverbal IQ } \\
\text { (Raven's, IQ norms) }\end{array}$ & $105.3(13.1)$ & $102.6(11.9)$ & $108.5(11.1)$ & 1.3 & .28 & .03 \\
\hline $\begin{array}{l}\text { Receptive vocabulary } \\
\text { (PPVT-III, standard scores) }\end{array}$ & $108.7(16.4)$ & $104.9(23.0)$ & $116.6(9.7)$ & $3.6^{2}$ & .03 & .09 \\
\hline \multicolumn{7}{|l|}{ Visual acuity } \\
\hline LogMAR & $-0.33(-0.84)$ & $-0.28(-0.85)$ & $-0.30(-0.80)$ & 1.3 & .25 & .02 \\
\hline Decimal & 0.47 & 0.52 & 0.49 & & & \\
\hline
\end{tabular}

AUT Autism, AS Asperger syndrome, AA Atypical autism/PDD-NOS, SPM standard progressive matrices, $P P V T$ peabody picture vocabulary test

* ANCOVA adjusted for age, IQ, sex, nonverbal IQ and receptive vocabulary

${ }^{1} \mathrm{SCH}>\operatorname{ASD} / \mathrm{TYP}(p \leq .03),{ }^{2} \mathrm{TYP}>\mathrm{SCH}(p=.049)$ (post-hoc Scheffé tests) 
ADOS autism spectrum cut-off was met. A diagnosis of atypical autism/PDD-NOS was assigned, if two domain cut-offs were met in the ADI-R (one of which was the social interaction domain) and ADOS autism spectrum cut-off was met. Autism, Asperger syndrome and atypical autism/PDD-NOS participants were pooled, as, owing to a rich body of evidence, the spectrum concept will replace the aforementioned single diagnosis in the upcoming DSM-V. About half of the participants with ASD were medication free, while the remainder received either risperidone, methylphenidate, atomoxetine, SSRIs or thyroxin. The majority of $\mathrm{SCH}$ probands received atypical antipsychotics. There is little research on how all these drugs influence VA. Nevertheless, the existing data for antipsychotics indicate that they do not affect VA or rather normalize sensory processing, respectively (Chen et al. 2003; Keedy et al. 2009). SCH diagnoses were endorsed by scores on the Positive and Negative Syndrome Scale (PANSS) (Kay et al. 1987). TYP had to be in the non-clinical range $(\mathrm{T}<68)$ of the Youth Self Report (YSR) and the parent rated Child Behavior Checklist (CBCL), the Young Adult Self Report (YASR), and the parent rated Young Adult Behavior Checklist (YABC) (Achenbach and Rescorla 2001, 2003), respectively. The groups were comparable with regard to sex distribution $\left[\chi^{2}(2)=2.7, p=.31\right]$, handedness (Edinburgh Handedness Inventory) (Oldfield 1971) $\left[\chi^{2}(4)=\right.$ 3.6, $p=.50]$, and nonverbal IQ (Raven's SPM) (Kratzmeier and Horn 1987) $[F(2,75)=1.3, p=.28$, $\left.\eta^{2}=.03\right]$. Age $\left[F(2,75)=6.6, p=.004, \eta^{2}=14\right)$ and receptive vocabulary (Peabody Picture Vocabulary TestIII) (Bulheller and Häcker 2004) $[F(2,75)=3.6, p=.03$, $\left.\eta^{2}=.09\right)$ differed between groups, as participants with $\mathrm{SCH}$ were older than those with ASD $(p=.004)$ and TYP ( $p=.03)$, and TYP had better receptive vocabulary than SCH $(p=.049)$. ASD and TYP did not differ on age and receptive vocabulary $\left(p_{\text {all }}>.10\right)$. All participants were recruited within an ongoing functional neuroimaging study on ASD versus SCH, where the FrACT was assessed routinely together with perimetry and form discrimination to determine exclusion/inclusion criteria. Clinical participants were inpatients or outpatients of the author's and neighboring psychiatric departments. TYP were recruited from local schools or by personal contact. As the current analysis of the FrACT data is retrospective in nature (VA testing was part of a standard intake evaluation), VA assessments were made independently of the study's research question, and authors did not have an a priori hypothesis about the findings. All included participants had reported normal or corrected vision. Procedures had been approved by the local ethical committee, and participants gave informed consent.
Visual Acuity Measure

As in the study by Ashwin et al., the FrACT (version 3.0.4) was used to assess visual acuity, which has demonstrated high test-retest reliability. It is a standardized automatic procedure using Landolt-Cs that can be presented on a screen of any state-of-art computer. Landolt-Cs are shown in up to eight different positions. Testees have to decide where the gap position is located and press appropriate buttons on a keyboard. PEST (Best Probability Estimation of Sensory Threshold) is used to estimate VA. VA thresholds are calculated depending on viewing distance and can be expressed as decimal acuity. Decimal acuity values are non-normally distributed. If parametric statistics are applied on decimal VA values these have to be logarithmized first. Thus, VA values can also be expressed as logMAR (logarithm of the Minimum Angle of Resolution). These values meet the assumptions of arithmetic and parametric procedures, but are less easy understood, as for instance decreasing values indicate better vision.

In the Ashwin et al. (2009a) study, the ASD group had a mean decimal acuity of 2.79, and TYP of 1.44 after values had been extrapolated using "post-hoc maximum likelihood analysis" in FrACT. Contrary to Ashwin et al., and except for the markedly reduced observation distance, which was set at $50 \mathrm{~cm}(60 \mathrm{~cm}$ in Ashwin et al. 2009a; default is $4 \mathrm{~m}$ ), all defaults of the FrACT were maintained in this study: number of trials (24), threshold definition: DIN/ISO corrected, time-out value: 30 s., post-hoc maximum likelihood analysis: "off", number of optotype directions: 8). Viewing distance was reduced as participants were sitting at a computer screen, where they also performed other tests in order to keep social demands to a minimum and shape a homogeneous testing phase. For FrACT administration participants were instructed to identify the location of the Landolt $\mathrm{C}$ gap and press the corresponding key on the keyboard with their right hand. The monitors used [CRT, 17" $(43.1 \mathrm{~cm}), 1024 \times 768$ pixels, $85 \mathrm{~Hz}$ ] yielded a maximum decimal VA of 0.89 (logMAR $=-0.06)$ on the FrACT program, substantially higher than in Ashwin et al. (2009a), but also restricted in assessing high VA thresholds. Reported FrACT decimal VA values in this study are unmodified raw scores [the post-hoc maximum likelihood analysis was not activated] and thus generally far lower than the extrapolated values reported in the Ashwin et al. (2009a) study.

\section{Results}

LogMAR values were used for statistics [mean decimal acuity values are also occasionally provided to improve 
understandability and comparability with Ashwin et al.]. Shapiro-Wilk's Normality Test showed that LogMAR values were normally distributed within the groups ( $w=.88$ to $.91, p<.01)$, and Levene's Test homogeneity of variances $(F<1.53, p>.22)$. Please note again that compared to decimal acuity increasing $\log$ MAR values correspond to lower visual acuity. Thus negative correlations of other parameters with $\log$ MAR indicate a positive association with VA (see Table 2). Neither age $(r=.13$, $p=.27$ ) (confidence interval [CI, 95\%] $=-.21$ to .24 ) nor receptive vocabulary $(r=.06, p=.60)(\mathrm{CI}=-.17$ to .23) correlated (Pearson's) substantially with VA in the total sample. Nevertheless, although age did not correlate significantly within VA in all groups, interestingly, the age/ VA correlation differed significantly between groups $\left(\chi^{2}=15.4, d f=2, p<.05\right)$ : it was $r=-.20$ (n.s.) in the ASD (CI $=-.38$ to .32$)$, but $r=.19$ (n.s.) $(\mathrm{CI}=-.37$ to $.45)$ in TYP and $r=.38(p=.04)(\mathrm{CI}=-.44$ to .65$)$ in SCH sample. IQ $(r=-.25)(\mathrm{CI}=-.20$ to .26$)$ correlated slightly, but significantly $(p=.03)$ with VA in the total sample. This correlation was significant for ASD and TYP, but not for $\mathrm{SCH}$.

Assuming the huge effect found by Ashwin et al. (2009a) ( $>4$ SDs), an $\alpha$ of .05 and the present sample size $(N=76)$, comparing three groups in a general linear model (SPSS/win, 19.) the power (1- $\beta$ ) of the current study to detect the previously reported effect was close to $100 \%$. It was $>90 \%$ for any other large (Cohen's $f>.40$; G-Power, 2.0), and even robust for medium effect $(f=.25$, $1-\beta=.51)$. However, as the present study did not predict VA differences between the groups (null hypothesis), equivalence testing (using ACOMED, version 2) was also applied to examine non-difference between groups.

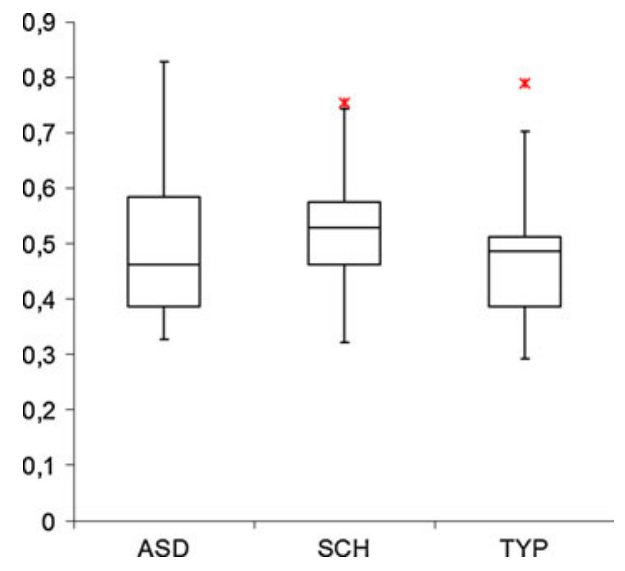

Fig. 1 Box-Plot for decimal VA in ASD, SCH and TYP

We found the mean $\operatorname{logMAR}$ as $-0.33(\mathrm{SD} \pm 0.84)$ (corresponding to a $M$ decimal VA $=0.47$ ) in the ASD group, -0.28 in $\mathrm{SCH}(\mathrm{SD} \pm 0.85)(M$ decimal $\mathrm{VA}=$ $0.52)$, and $-0.30(\mathrm{SD} \pm 0.80)(M$ decimal VA $=0.49)$ in TYP (see Table 1; Fig. 1). Values for all groups showed unimodal distributions. A one-way ANCOVA was computed to compare VA between groups, with the factors group (ASD, SCH, TYP), sex (male, female), and medication (any medication, no medication) as well as age, nonverbal IQ and receptive vocabulary as covariates to adjust for possibly remaining confounding effects. ANCOVA did not reveal a significant main effect for group $\left[F(2,75)=1.4, p=.27, \eta^{2}=.02\right]$, sex $[F(1,75)=.32$, $\left.p=.78, \eta^{2}=.00\right]$, medication $[F(1,75)=.73, p=.62$, $\left.\eta^{2}=.00\right]$, a group-by-sex interaction $[F(2,75)=0.55$, $\left.p=.59, \eta^{2}=.02\right]$ or any higher interaction effect. A separate ANCOVA for the ASD subsample sample alone comparing logMAR between ASD subgroups: autism
Table 2 Pearson correlations between visual acuity (VA), biological, cognitive and clinical variables in Autism Spectrum Disorders (ASD), Schizophrenia (SCH), typically developing (TYP) and total sample [note that negative correlations indicate a positive association with VA, due to the usage of $\log \mathrm{MAR}]$

$* p<.05, A D I-R$ Autism diagnostic interview-revised, ADOS autism diagnostic observation schedule, PANSS positive and negative syndrome scale

\begin{tabular}{|c|c|c|c|c|}
\hline Demographic/clinical variables & $\begin{array}{l}\text { ASD } \\
\text { VA }\end{array}$ & $\begin{array}{l}\mathrm{SCH} \\
\mathrm{VA}\end{array}$ & $\begin{array}{l}\text { TYP } \\
\text { VA }\end{array}$ & $\begin{array}{l}\text { Total sample } \\
\text { VA }\end{array}$ \\
\hline Age & -.20 & $.38^{*}$ & .19 & .13 \\
\hline Nonverbal IQ (Raven's SPM) & $-.28 *$ & .07 & $-.37 *$ & $-.25^{*}$ \\
\hline Receptive vocabulary (PPVT-III) & -.09 & -.31 & .01 & -.05 \\
\hline \multicolumn{5}{|l|}{ PANSS } \\
\hline Positive & - & -.02 & & \\
\hline Negative & - & -.03 & & \\
\hline \multicolumn{5}{|l|}{ ADI-R } \\
\hline Social & -.08 & & & \\
\hline Communication & -.16 & & & \\
\hline Stereotypies & -.11 & & & \\
\hline \multicolumn{5}{|l|}{ ADOS } \\
\hline Social & .12 & & & \\
\hline Communication & -.03 & & & \\
\hline Stereotypies & .22 & & & \\
\hline
\end{tabular}


$(M=-0.31, \mathrm{SD} \pm 0.83)(M$ decimal VA $=0.49)$, Asperger syndrome $(M=-0.32, \quad \mathrm{SD} \pm 0.86) \quad(M$ decimal $\mathrm{VA}=0.48)$, atypical autism/PDD-NOS $(M=-0.35$, $\mathrm{SD} \pm 0.77)(M$ decimal VA $=0.45)$ did not show any differences $\left[F(2,26)=.44, p=.64, \eta^{2}=.03\right)$. Testing for equivalence ( $\delta=0.2, \alpha=5 \%$ ) confirmed non-different VA results for ASD versus TYP, SCH versus TYP, and ASD subgroups (interval $<0.16$ ), but not for ASD versus $\mathrm{SCH}$ (interval $=0.26$ ). There were no significant correlations $(p>.22)$ between VA and the subscales of the ADOS/ADI$\mathrm{R}$ in ASD $(r=.22$ to -.15$)$ or the subscales of the PANSS in $\mathrm{SCH}(r=-.02$ and -.03$)$ (see Table 2$)$.

\section{Conclusions}

The present study could not confirm the eagle-eyed VA hypothesis of autism as postulated by Ashwin et al. (2009a, b). VA in ASD was consistent with the one measured in TYP and SCH when using a short viewing distance, the default number of trials and no "post-hoc maximum likelihood analysis" in FrACT. Moreover, we did not find sex differences in terms of males outperforming females, as might be expected by the systemizing/empathizing theory of gender differences and hypersystemizing approach to ASD (Baron-Cohen et al. 2009). Indeed, as females with ASD are often more severely affected by core and peripheral symptoms than males, the opposite prediction might be similarly justified. In accord with Ashwin et al., ASD subgroups (autism, Asperger's syndrome, atypical autism/ PDD-NOS) did not differ on VA, excluding the possibility of eagle-eyed vision being a valid phenomenon for a more circumscribed clinical picture of ASD. It seems that the previously reported results which lead to the hypothesis of eagle-eyed VA in ASD may indeed be based on a cascade of disadvantageous events owing to changes in the FrACT default settings as suspected by Bach and Dakin (2009) and Crewther and Sutherland (2009), particularly the combination of short viewing distance, high amount of trials and extrapolation of results.

The present study also suffers from methodological limitations when using the FrACT: the viewing distancelike in Ashwin et al., was chosen low, so that visual acuity values are prone to ceiling effects. The size of the Landolt $\mathrm{C}$ needs to be reduced below the VA threshold value to adequately measure VA, but the optotype size in both studies did not get close enough to this threshold to do so. Thus, authentic measurement of "eagle-eye" levels of visual acuity was hampered, and there remain doubts that if it were possible to measure higher levels of VA a difference would be found. However, VA in our study was well below 1.0 in the three groups, respectively, and distribution and variability of VA values within and between groups did not indicate any ceiling effect. Hence, the risk for biased results in this study appears small. Meanwhile, a study by Kéita et al. (2010) also failed to demonstrate visual hyperacuity to Landolt-C optotypes, whether defined by luminance- or texture-contrast, and an ongoing replication study by the members of the Baron-Cohen group themselves in adults with ASD also points to no evidence for visual hyperacuity (Tavassoli et al. 2010). This is perhaps not surprising, as previous optometric vision research in ASD has either observed comparable visual function to TYP, or even visual impairment (e.g. strabismus, oculomotor difficulties) in ASD (see Trachtman 2008; Simmons et al. 2009, for recent reviews).

On balance, like in Ashwin et al. (2009a), age correlated low to moderate and negative with VA in TYP and $\mathrm{SCH}$, but positive in ASD. Although many of these correlations were not significant, differences in correlation were significant between $\mathrm{ASD}$ and $\mathrm{SCH}$ and there was a trend between ASD and TYP, indicating a potential disparity in VA between groups with mounting age. We analyzed a markedly younger sample than Ashwin et al. (2009a), and perhaps this might also account for some of our negative findings. Although highly speculative and appearing paradoxical, superior visual acuity could be a creep-in phenomenon of later adulthood in ASD, with no or less typical age related decline in ASD, or markedly delayed visual maturation, respectively (Goodman and Ashby 1990). Hypersensitivity might be viewed a compensatory process not having taken place until later adulthood. Consistently, a recent study demonstrated tactile hypersensitivity in adults with Asperger syndrome (Blakemore et al. 2006). In general, detecting hypersensitivity in ASD might be closely related to study design. Depending on the modality (visual, auditory, tactile), methodology (self-report, sensory measures), and sample (age, IQ, gender, ASD diagnosis) one might detect autistic superiority or not. Overall, hyporesponsiveness to sensory stimuli has still been more frequently observed in ASD than hypersensitivity (Rogers and Ozonoff 2005).

In sum, the present study did not find evidence to support the eagle-eyed VA hypothesis of ASD. Future research on hypersensitivity in ASD will need to investigate processes across sensory modalities and methodologies in order to determine which autistic individuals are hypersensitive to which stimuli. Hereby, it seems particularly important to differentiate between scientific experimental studies in the field of cognitive neuroscience of perception in ASD, on one hand, and more unspecific studies on experienced hypersensitivity by individuals with ASD using questionnaire/interview and anecdotal information (Davis et al. 2006; Minshew and Hobson 2008), on the other. 
Acknowledgments The authors sincerely thank Prof. Michael Bach for guidance in using the FrACT and Prof. Simon Baron-Cohen, Dr. Chris Ashwin, and Teresa Tavassoli for valuable discussion.

Open Access This article is distributed under the terms of the Creative Commons Attribution Noncommercial License which permits any noncommercial use, distribution, and reproduction in any medium, provided the original author(s) and source are credited.

\section{References}

Achenbach, T. M., \& Rescorla, L. A. (2001). Manual for ASEBA school-age forms \& profiles. Burlington, VT: University of Vermont, Research Center for Children, Youth \& Families.

Achenbach, T. M., \& Rescorla, L. A. (2003). Manual for ASEBA adult forms \& profiles. Burlington, VT: University of Vermont, Research Center for Children, Youth \& Families.

Ashwin, E., Ashwin, C., Rhydderch, D., Howells, J., \& Baron-Cohen, S. (2009a). Eagle-eyed visual acuity: An experimental investigation of enhanced perception in autism. Biological Psychiatry, $65,17-21$.

Ashwin, E., Ashwin, C., Tavassoli, T., Chakrabarti, B., \& BaronCohen, S. (2009b). In reply to Commentary by Bach et al.: Eagle-eyed visual acuity in autism. Biological Psychiatry, 66, e23-e24.

Ashwin, C., Ashwin, E., Tavassoli, T., Howells, J., Rhydderch, D., \& Baron-Cohen, S. (2009c). Olfactory hypersensitivity in autism spectrum conditions (submitted).

Bach, M. (1996). The Freiburg Visual Acuity test (FrACT)-automatic measurement of visual acuity. Optometry and Vision Science, 73, 49-53. [FrACT is available at: http://www.michaelbach.de/fract/ index.html. Accessed February 24, 2010].

Bach, M., \& Dakin, S. C. (2009). Regarding "Eagle-eyed visual acuity: An experimental investigation of enhanced perception in autism”. Biological Psychiatry, 66, e19-e20.

Baron-Cohen, S., Ashwin, E., Ashwin, C., Tavassoli, T., \& Chakrabarti, B. (2009). Talent in autism: Hyper-systemizing, hyper-attention to detail and sensory hypersensitivity. Philosophical Transactions of the Royal Society of London. Series B, Biological Sciences, 27, 1377-1383.

Belmonte, M. K., \& Yurgelun-Todd, D. A. (2003). Functional anatomy of impaired selective attention and compensatory processing in autism. Brain Research Cognitive Brain Research, 17, 651-664

Bertone, A., Mottron, L., Jelenic, P., \& Faubert, J. (2005). Enhanced and diminished visuo-spatial information processing in autism depends on stimulus complexity. Brain, 128, 2430-2441.

Blakemore, S. J., Tavassoli, T., Calò, S., Thomas, R. M., Catmur, C., Frith, U., et al. (2006). Tactile sensitivity in Asperger syndrome. Brain and Cognition, 61, 5-13.

Bölte, S., Holtmann, M., Poustka, F., Scheurich, A., \& Schmidt, L. (2007). Gestalt perception and local-global processing in highfunctioning autism. Journal of Autism and Developmental Disorders, 37, 1493-1504.

Bölte, S., Rühl, D., Schmötzer, G., \& Poustka, F. (2006). Diagnostisches Interview für Autismus-Revidiert (ADI-R). Bern: Huber.

Bonnel, A., Mottron, L., Peretz, I., Trudel, M., Gallun, E., \& Bonnel, A. M. (2003). Enhanced pitch sensitivity in individuals with autism: A signal detection analysis. Journal of Cognitive Neuroscience, 15, 226-235.

Bulheller, S., \& Häcker, H. (2004). Peadbody picture vocabulary test $(P P V T)$. Frankfurt/M: Harcourt.
Caron, M. J., Mottron, L., Berthiaume, C., \& Dawson, M. (2006). Cognitive mechanisms, specificity and neural underpinnings of visuospatial peaks in autism. Brain, 129, 1789-1802.

Chen, Y., Levy, D. L., Sheremata, S., Nakayama, K., Matthysse, S., \& Holzman, P. S. (2003). Effects of typical, atypical, and no antipsychotic drugs on visual contrast detection in schizophrenia. American Journal of Psychiatry, 160, 1795-1801.

Couture, S. M., Penn, D. L., Losh, M., Adolphs, R., Hurley, R., \& Piven, J. (2010). Comparison of social cognitive functioning in schizophrenia and high functioning autism: More convergence than divergence. Psychological Medicine, 40, 569-579.

Crewther, D. P., \& Sutherland, A. (2009). The more he looked inside, the more piglet wasn't there: Is autism really blessed with visual hyperacuity? Biological Psychiatry, 66, e21-e22.

Dakin, S., \& Frith, U. (2005). Vagaries of visual perception in autism. Neuron, 48, 497-507.

Davis, R. A., Bockbrader, M. A., Murphy, R. R., Hetrick, W. P., \& O’Donnell, B. F. (2006). Subjective perceptual distortions and visual dysfunction in children with autism. Journal of Autism and Developmental Disorders, 36, 199-210.

Geschwind, D. H., \& Levitt, P. (2007). Autism spectrum disorders: Developmental disconnection syndromes. Current Opinion in Neurobiology, 17, 103-111.

Goodman, R., \& Ashby, L. (1990). Delayed visual maturation and autism. Developmental Medicine and Child Neurology, 32, $814-819$

Grandin, T. (2000). My experiences with visual thinking, sensory problems and communication difficulties. http://www.autism.com/ ind_temple_experiences.asp .Last retrieval 14th April, 2011.

Greenaway, R., \& Plaisted, K. (2005). Top-down attentional modulation in autistic spectrum disorders is stimulus-specific. Psychological Science, 16, 987-994.

Guilmatre, A., Dubourg, C., Mosca, A. L., Legallic, S., Goldenberg, A., Drouin-Garraud, V., et al. (2009). Recurrent rearrangements in synaptic and neurodevelopmental genes and shared biologic pathways in schizophrenia, autism, and mental retardation. Archives of General Psychiatry, 66, 947-956.

Hancock, P. J., Walton, L., Mitchell, G., Plenderleith, Y., \& Phillips, W. A. (2008). Segregation by onset asynchrony. Journal of Vision, 8, 21.1-2121.

Happé, F. G. (1996). Studying weak central coherence at low levels: Children with autism do not succumb to visual illusions. A research note. Journal of Child Psychology and Psychiatry, 37, 873-877.

Happé, F. G., \& Frith, U. (2006). The weak coherence account: Detail-focused cognitive style in autism spectrum disorders. Journal of Autism and Developmental Disorders, 36, 5-25.

Heaton, P., Davis, R. E., \& Happé, F. G. (2008). Research note: Exceptional absolute pitch perception for spoken words in an able adult with autism. Neuropsychologia, 46, 2095-2098.

Hill, E. L., \& Frith, U. (2003). Understanding autism: Insights from mind and brain. Philosophical Transactions of the Royal Society of London. Series B, Biological Sciences, 358, 281-289.

Johnson, S. C., Lowery, N., Kohler, C., \& Turetsky, B. I. (2005). Global-local visual processing in schizophrenia: Evidence for an early visual processing deficit. Biological Psychiatry, 58, 937-946.

Jolliffe, T., \& Baron-Cohen, S. (1999). A test of central coherence theory: Linguistic processing in high-functioning adults with autism or Asperger syndrome: Is local coherence impaired? Cognition, 71, 149-185.

Kay, S. R., Fiszbein, A., \& Opler, L. A. (1987). The positive and negative syndrome scale (PANSS) for schizophrenia. Schizophrenia Bulletin, 13, 261-276.

Keedy, S. K., Rosen, C., Khine, T., Rajarethinam, R., Janicak, P. G., \& Sweeney, J. A. (2009). An fMRI study of visual attention and 
sensorimotor function before and after antipsychotic treatment in first-episode schizophrenia. Psychiatry Research, 172, 16-23.

Kéita, L., Mottron, L., \& Bertone, A. (2010). Far visual acuity is unremarkable in autism: Do we need to focus on crowding? Autism Research, 3, 333-341.

Kolvin, I. (1971). Studies in childhood psychosis. I. Diagnostic criteria and classification. British Journal of Psychiatry, 118, 381-384.

Kratzmeier, H., \& Horn, R. (1987). Raven-matrizen-test (SPM). Göttingen: Beltz.

Mackinlay, R., Charman, T., \& Karmiloff-Smith, A. (2006). High functioning children with autism spectrum disorder: A novel test of multitasking. Brain and Cognition, 61, 14-24.

Magnée, M. J., Oranje, B., van Engeland, H., Kahn, R. S., \& Kemner, C. (2009). Cross-sensory gating in schizophrenia and autism spectrum disorder: EEG evidence for impaired brain connectivity? Neuropsychologia, 47, 1728-1732.

Minshew, N. J., \& Hobson, J. A. (2008). Sensory sensitivities and performance on sensory perceptual tasks in high-functioning individuals with autism. Journal of Autism and Developmental Disorders, 38, 1485-1498.

Mottron, L., Dawson, M., Soulières, I., Hubert, B., \& Burack, J. (2006). Enhanced perceptual functioning in autism: an update, and eight principles of autistic perception. Journal of Autism and Developmental Disorders, 36, 27-43.

Neumann, D., Spezio, M. L., Piven, J., \& Adolphs, R. (2006). Looking you in the mouth: Abnormal gaze in autism resulting from impaired top-down modulation of visual attention. Social Cognition and Affective Neuroscience, 1, 194-202.

Oldfield, R. C. (1971). The assessment and analysis of handedness: The Edinburgh inventory. Neuropsychologia, 9, 97-113.

Plaisted, K. (2001). Reduced generalization in autism: An alternative to weak central coherence. In: J. A. Burack, T. Charman, N. Yirmiya, \& P. R. Zelazo (Eds.), The development of autism: Perspectives from theory and research (pp. 149-169). Mahwah, NJ: Erlbaum.

Pring, L. (2005). Savant talent. Developmental Medicine and Child Neurology, 47, 500-503.

Rapoport, J., Chavez, A., Greenstein, D., Addington, A., \& Gogtay, N. (2009). Autism spectrum disorders and childhood-onset schizophrenia: Clinical and biological contributions to a relation revisited. Journal of the American Academy of Child and Adolescent Psychiatry, 48, 10-18.

Rassow, B., Cavazos, H., \& Wesemann, W. (1990). Normgerechte Sehschärfenbestimmung mit Buchstaben. Augenärztliche Fortbildung, 13, 105-114.

Ribolsi, M., Koch, G., Magni, V., Di Lorenzo, G., Rubino, I. A., Siracusano, A., et al. (2009). Abnormal brain lateralization and connectivity in schizophrenia. Review in the Neurosciences, 20, 61-70.

Rogers, S. J., \& Ozonoff, S. (2005). Annotation: What do we know about sensory dysfunction in autism? A critical review of the empirical evidence. Journal of Child Psychology and Psychiatry, $46,1255-1268$.

Rühl, D., Bölte, S., Feineis-Matthews, S., \& Poustka, F. (2004). Diagnostische Beobachtungsskala für Autistische Störungen (ADOS). Bern: Huber.

Seltzer, M. M., Abbeduto, L., Krauss, M. W., Greenberg, J., \& Swe, A. (2004). Comparison groups in autism family research: Down syndrome, fragile $\times$ syndrome, and schizophrenia. Journal of Autism and Developmental Disorders, 34, 41-48.

Shah, A., \& Frith, U. (1983). An islet of ability in autistic children: A research note. Journal of Child Psychology and Psychiatry, 24, 613-620.

Simmons, D. R., Robertson, A. E., McKay, L. S., Toal, E., McAleer, P., \& Pollick, F. E. (2009). Vision in autism spectrum disorders. Vision Research, 49, 2705-2739.

Sporn, A. L., Addington, A. M., Gogtay, N., Ordoñez, A. E., Gornick, M., Clasen, L., et al. (2004). Pervasive developmental disorder and childhood-onset schizophrenia: Comorbid disorder or a phenotypic variant of a very early onset illness? Biological Psychiatry, 55, 989-994.

Sutherland, A., \& Crewther, D. P. (2010). Magnocellular visual evoked potential delay with high autism spectrum quotient yields a neural mechanism for altered perception. Brain, 133, 20892097.

Tavassoli, T., Ashwin, E., Ashwin, C., Chakrabarti, B., \& BaronCohen, S. (2009). Tactile and auditory hypersensitivity in autism spectrum conditions (submitted).

Tavassoli, T., Latham, K., \& Baron-Cohen, S. (2010). Visual processing in autism spectrum conditions. Poster presented at the 3rd conference of the Scientific Society Autism Spectrum (WTAS), 18th-19th February, Frankfurt/M., Germany.

Tomchek, S. D., \& Dunn, W. (2007). Sensory processing in children with and without autism: A comparative study using the short sensory profile. American Journal of Occupational Therapy, 61, 190-200.

Trachtman, J. N. (2008). Background and history of autism in relation to vision care. Optometry, 79, 391-396.

Yeap, S., Kelly, S. P., Sehatpour, P., Magno, E., Javitt, D. C., Garavan, H., et al. (2006). Early visual sensory deficits as endophenotypes for schizophrenia: High-density electrical mapping in clinically unaffected first-degree relatives. Archives of General Psychiatry, 63, 1180-1188. 